\title{
Evaluation de l'effet trophique de l'huile de neem (Azadirachta indica) chez les poulets de chair de souche Sasso
}

\author{
Komi KOMI KOUKOURA ${ }^{1 *}$, Marc BRAH ${ }^{1,2}$, Yendoubé T. KANTATI ${ }^{3}$ et \\ Tchadjobo TCHACONDO ${ }^{1}$
}

\author{
${ }^{1}$ Laboratoire des Sciences Biomédicales, Alimentaires et Santé Environnementale (LaSBASE), Ecole \\ Supérieure des Techniques Biologiques et Alimentaires (ESTBA), Université de Lomé, Togo. \\ ${ }^{2}$ Centre d'Excellence Régional sur les Sciences Aviaires (CERSA), Université de Lomé, Togo. \\ ${ }^{3}$ Département de physiologie animale: Laboratoire de Physiologie et de Pharmacologie, Unité de Recherche: \\ Physiopathologie, Substances Bioactives et Sécurité. Faculté des Sciences, université de Lomé, Togo. \\ *Auteur correspondant ; E-mail : kkoufrede@outlook.fr
}

\section{REMERCIEMENTS}

Nos sincères remerciements à la Banque Mondiale pour le financement de ce projet de recherche à travers le Centre d'Excellence Régional sur les Sciences Aviaires (CERSA) de l'Université de Lomé, République du Togo.

\author{
Received: 27-06-2021 $\quad$ Accepted: 29-11-2021 $\quad$ Published: 31-12-2021
}

\section{RESUME}

L'alimentation, facteur environnemental déterminant est indispensable pour une production avicole de qualité. Face aux contraintes alimentaires, différentes matières premières non conventionnelles ont été introduites dans les rations de la volaille avec l'usage des matières grasses. Le but de cette étude a été d'évaluer l'effet de l'huile de neem sur les performances zootechniques, les caractéristiques de la carcasse et les paramètres biochimiques sériques des poulets de chair à croissance lente ; par la méthode d'alimentation d'un lot test avec de la nourriture contenant de l'huile de neem, par rapport à un lot témoin avec de la nourriture dépourvue de l'huile de neem. Jusqu'à cinq semaines d'âge de traitement avec des concentrations respectives de 0,25\% (T0,25), $0,50 \%(\mathrm{~T} 0,50), 0,75 \%(\mathrm{~T} 0,75)$ et $1 \%$ (T1), les individus du lot $\mathrm{T} 0,50$ ont montré la meilleur Indice de Consommation et un gain considérable de poids par rapport aux autres lots et au lot témoin. Il a été aussi démontré que, les poulets traités ont présenté une longueur de l'intestin plus élevée et un poids du cœur plus faible que les témoins. L'alimentation des volailles avec de la nourriture à base d'huile de neem auraient donc un effet bénéfique sur les performances de production. Ces résultats pourraient apporter une contribution à l'amélioration de la production des poulets de chair.

(C) 2021 International Formulae Group. All rights reserved.

Mots clés : Huile de neem, performances zootechniques, carcasse, paramètres biochimiques.

\section{Evaluation of the trophic effect of neem oil (Azadirachta indica) in Sasso strain broilers}

\begin{abstract}
Feed, a determining environmental factor, is essential for quality poultry production. Due to dietary constraints, different non-conventional raw materials have been introduced in poultry rations with the use of fats. The aim of this study was to evaluate the effect of neem oil on zootechnical performance, carcass characteristics,
\end{abstract}


and serum biochemical parameters of slow growing broilers; by feeding a test batch with feed containing neem oil, compared to a control batch with feed without neem oil. Five week chicks were given neem oil feed with concentrations of 0.25 (T0.25), 0.50 (T0.50), 0.75 (T0.75), and 1\% (T1), respectively. The individuals of the T0.50 batch showed the best Consumption Index and a considerable weight gain compared to the other batches and the control batch. The treated chickens also showed an increase gut length and a decrease heart weight than those of the controls. Feeding chickens with neem oil feed showed a beneficial effect on production performance. These results could contribute to the improvement of broiler production.

(C) 2021 International Formulae Group. All rights reserved.

Keywords: Neem oil, zootechnical performance, carcass, biochemical parameters.

\section{INTRODUCTION}

En Afrique, l'élevage est une activité cosmopolite. La production avicole a connu une croissance des plus fulgurantes en Afrique de l'ouest avec un effectif total de volailles qui est passé de 290 millions de têtes en 2000 à 730 millions de têtes en 2017(FAO, 2018). Au Togo, l'effectif total de volailles estimé à 8,8 millions de têtes en 2010 est passé à 26 millions de têtes en 2019 (FAO, 2020), soit un taux de progression de près de $200 \%$. Compte tenu de son prix abordable, de l'absence d'interdit religieux à son encontre et de ses qualités nutritionnelles, la volaille occupe une place de choix dans le menu des ménages. La courte durée du cycle d'élevage des volailles (poulets) facilite également leur production (Sonaiya et Swan, 2004 ; Nahimana et al, 2019).

Cependant, l'aviculture est sujette à des contraintes diverses telles que : la précarité des conditions d'habitat et d'hygiène, l'absence de prophylaxie, la faiblesse de la formation, de l'information et de la sensibilisation des producteurs et enfin l'insuffisance d'une alimentation quantitative et qualitative (Tossou et al., 2014). En effet l'alimentation occupe une place centrale au sein de ces facteurs susmentionnés. En aviculture, l'alimentation représente 70 à $80 \%$ des coûts de production et joue un rôle prépondérant sur les performances et les qualités des produits (Sonaiya et Swan, 2004 ; Ponka et al., 2016 ; Inoussa et al., 2020). Afin de remédier à cette contrainte, des études ont été menées pour valoriser de nouvelles ressources végétales de haute valeur nutritive non marchandes et localement disponibles
(Novidzro et al., 2019) pour nourrir la volaille. Ainsi, les extraits de végétaux tels que les huiles essentielles, les huiles végétales extraites des graines de plantes ont été proposées afin d'améliorer la productivité et la qualité des produits alimentaires issus des animaux (Windisch et al., 2008). Le neem, (Azadirachta indica A. Juss), est une plante de la famille des Meliaceae, largement diffusé en Afrique (Koona et Budida 2011 ; Ogbuewu et al. 2011; Diedhiou, 2017). Cette plante est bien adaptée aux conditions climatiques et édaphiques des forêts tropicales humides et du Sahel (Vanessa et al., 2019). Les études ont montré que la poudre des feuilles de neem à 2,5 $\mathrm{g} / \mathrm{kg}$ d'aliments a des effets très bénéfiques sur la croissance et les paramètres hématobiochimiques des poulets de chair (Jahanzeb et al., 2012). Par ailleurs, l'huile extraite des graines de neem contient du triterpénoïde, des composés tels que l'azadirachtine, la gédunine, la nimbine et la nimbidine qui ont des propriétés antibactériennes et antifongiques (Makeri et al., 2007; Valarmathy et al., 2010). Ces composés contenus dans l'huile de neem, une fois incorporés dans l'alimentation des volailles, peuvent produire des effets additifs ou synergiques sur les performances de production (Valarmathy et al., 2010). Cette étude s'était inscrite dans cette dynamique et avait eu pour objectif, de contribuer à l'amélioration de la productivité des poulets de chair à croissance lente de souche Sasso par l'incorporation de l'huile de neem dans l'alimentation. 


\section{MATÉRIEL ET MÉTHODES \\ Cadre d'étude}

Cette étude a été réalisée à l'unité expérimentale du Centre d'Excellence Régional sur les Sciences Aviaires (CERSA) de l'Université de Lomé, Togo. L'Université de Lomé est située entre $6^{\circ} 10^{\prime} 38,5^{\prime \prime}$ de latitude nord et $1^{\circ} 12^{\prime} 46,8^{\prime \prime}$ de longitude Est. Cette position lui confère un climat de type subtropical avec deux saisons de pluies (la grande saison de pluie de fin mars ou avril à Juin et la petite saison de pluie de miseptembre à fin octobre) alternées de saisons sèches (la grande saison sèche de décembre à mars et la petite saison sèche de juillet à aout). La température ambiante est d'environ $27^{\circ} \mathrm{C}$, tandis que les précipitations annuelles oscillent entre 800 et $1400 \mathrm{~mm}$ (FAO, 2019).

\section{Matériel biologique}

Au total, 225 poussins Sasso âgés d'un jour, tous provenant du couvoir du CERSA, ont été élevés en bande unique sur de la litière de copeaux dans un environnement conventionnel (température ambiante de $31-33^{\circ} \mathrm{C}$ et une humidité relative de $45-70 \%$ ) jusqu'à cinq semaines d'âge.

\section{Conduite des animaux}

Dans cette expérimentation, la conduite des animaux a été faite en deux phases. La première phase a duré cinq semaines, allant de la réception des poussins d'un jour jusqu'à la fin du démarrage. La deuxième phase d'une durée de sept semaines, était comprise entre cinq semaines (l'âge à la fin du démarrage) et douze semaines (l'âge à l'abattage). Après cinq semaines d'élevage (période de démarrage), les poussins ont été répartis dans les loges pour la phase expérimentale (à partir de cinq semaines d'âge jusqu'à douze semaines d'âge). L'étude a été menée selon un plan entièrement randomisé avec cinq traitements. Chaque traitement a été répété trois fois, et comprenait 15 poulets de chair chacun. Le poids vif moyen des poulets de chair était de $300 \mathrm{~g}$. Les poussins ont été vaccinés selon le calendrier de vaccination standard. Pour réduire le stress causé par la vaccination aux oiseaux, 24 heures avant et après la vaccination, l'anti-stress Amintotal a été ajouté à l'eau de boisson. Le poulailler était équipé de mangeoires et d'abreuvoirs manuels ainsi que des loges grillagées. Les abreuvoirs étaient régulièrement lavés pour éviter les contaminations fécales et microbiennes.

\section{Matériel végétal}

L'huile végétale de neem était l'ingrédient expérimental qui a été utilisé au cours de cette étude. Elle a été fournie par une huilerie sise à Glazoué au Nord du Bénin, spécialisée dans l'extraction d'huile végétale. Un régime alimentaire en une seule phase (phase croissance-finition) a été adopté dans l'étude. Jusqu'à douze semaines (l'âge de l'abattage), les oiseaux ont reçu cinq régimes contenant différents taux d'incorporation d'huile de neem, formulés pour satisfaire les besoins en nutriments des poulets de chair. D'après les études de Diedhiou (2017) sur le fractionnement des graines de neem, la valeur moyenne du pouvoir calorifique de l'huile de neem était de 9477,18 kcal.kg-1 (39,61 MJ/kg). L'analyse de la composition en acides gras de l'huile de neem obtenue par Faye (2010) et Diedhiou (2017) a confirmé que l'huile de neem était majoritairement oléique (43\%), avec une proportion quasi équivalente d'acide palmitique $(17 \%)$, stéarique $(17 \%)$ et linoléique (19\%), et un rapport saturé/insaturé voisin de 0,5 traduisant le caractère nettement insaturé des huiles de neem. Toutefois ces compositions varient, par exemple en fonction de la période, de la zone de récolte et du matériel végétal.

\section{Régimes alimentaires expérimentaux}

Durant l'expérimentation, les traitements alimentaires étaient constitués comme suit: les poussins du lot témoin ont reçu l'aliment témoin (T0) dépourvu de l'huile de neem ; les poussins des lots T0,25 ; T0,50 ; 
$\mathrm{T} 0,75$ et $\mathrm{T} 1$ ont reçu respectivement l'aliment contenant 0,$25 ; 0,50 ; 0,75$ et $1 \%$ de l'huile de neem. Les ingrédients et composition nutritionnelle des différentes rations utilisées sont indiqués dans le Tableau 1. Tous les aliments expérimentaux étaient isoénergétiques et iso-protéiques. L'eau était servie ad libitum.

\section{Collecte des données}

Pour la collecte des données, les pesées ont été effectuées sur chaque poulet de façon hebdomadaires tandis que les quantités d'aliments distribuées dans les différents lots et les refus ont été pesées quotidiennement à l'aide d'une balance électronique de précision de marque CAMRY de sensibilité $1 \mathrm{~g}$. Ces données ont permis de déterminer les paramètres de production tels que : l'Ingestion Alimentaire (IA), le Gain de Poids (GP), le Poids Vif (PV) et l'Indice de Consommation (IC). A la onzième semaine d'essai, six poulets par traitements de poids moyen similaires ont été sélectionnés pour l'étude de digestibilité. Les poulets ont été disposés dans des cages équipées d'un abreuvoir à tétine et d'une mangeoire et ont été élevés pendant huit jours. Les poulets ont été pesés avant le début et à la fin de la phase de cette étude de digestibilité. Les quantités d'aliments servis et restants par jour ont été aussi pesées de même que les excréments. Un échantillon de $500 \mathrm{~g}$ a été prélevé, homogénéisé et séché à l'étuve pour les analyses au laboratoire en vue de déterminer les Coefficients d'Utilisation Digestive et Métabolique Apparent (CUDM) de la matière sèche et de la protéine. A la douzième semaine d'essai, neufs poulets par traitement ont été choisis au hasard et mis à jeun pour $24 \mathrm{~h}$ et ont été ensuite pesés. Ces poulets ont été abattus et éviscérés pour évaluer et enregistrer le rendement en carcasse et les différentes proportions d'organes.
Le rendement en carcasse et les différentes proportions d'organes ont été calculés et le résultat a été exprimé en pourcentage. Ainsi, les proportions du cœur, du pancréas, du gésier, du foie et de l'intestin ont été calculées. Les longueurs du duodénum, du jéjunum et de l'iléon ont été déterminées. Le muscle du bréchet a été prélevé en vue de la mesure du pH ultime. Des échantillons de sang des poulets abattus ont été prélevés dans des tubes secs et le sérum a été prélevé pour la détermination de la concentration sérique en glucose, en protéines totales, en albumine et en triglycérides. Le dosage de ces paramètres biochimiques a été réalisé au laboratoire du CERSA de l'Université de Lomé. Le taux sanguin du glucose a été déterminé par la méthode colorimétrique enzymatique avec le kit de dosage Cypress Diagnostics (Hulshout, Belgium). Les protéines totales ont été dosées par la méthode de Biuret décrite par Cypress Diagnostics (Hulshout, Belgium). L'albumine a été dosée par la méthode colorimétrique avec le kit de dosage Cypress Diagnostics (Hulshout, Belgium) et les triglycérides ont été dosés par spectrophotométrie colorimétrique avec le kit de dosage Cypress Diagnostics (Hulshout, Belgium).

\section{Analyse statistique}

Les données ont été saisies dans le tableur Excel® 2013 version 15.0 et le calcul des moyennes, des écarts-types, des variances a été effectué à l'aide du logiciel Graph Pad Prism 8.0.2 (GraphPad Software, San Diego, CA). La comparaison des moyennes a été faite par le test de Tukey. Le test non paramétrique de Kruskall-Wallis ou de Friedman a été utilisé pour les données qui n'ont pas suivi une distribution normale. Une valeur de $\mathrm{P}<0,05$ a été considérée comme significative. Les résultats sont présentés sous la forme de moyenne plus ou moins l'erreur standard de la moyenne $(\mathrm{M} \pm \mathrm{ESM})$. 
Tableau 1 : Ingrédients et composition nutritionnelle des différentes rations utilisées.

\begin{tabular}{lccccc}
\hline Type de matières premières & T0 & T0,25 & T0,50 & T0,75 & T1 \\
\hline Maïs $(\mathrm{kg})$ & 61,8 & 60,1 & 59,1 & 58 & 57,5 \\
Son cubé (kg) & 3,5 & 4,9 & 5,09 & 6,7 & 7 \\
Tourteau soja (kg) & 5,5 & 4,8 & 5 & 5 & 6 \\
Soja torréfié (kg) & 18,7 & 19,4 & 19 & 19 & 18 \\
Lysine (kg) & 0,2 & 0,2 & 0,2 & 0,2 & 0,2 \\
Méthionine (kg) & 0,1 & 0,1 & 0,1 & 0,1 & 0,1 \\
Coquille (kg) & 1 & 1 & 1 & 1 & 1 \\
Sel (kg) & 0,2 & 0,2 & 0,2 & 0,2 & 0,2 \\
Concentré chair 10\% ${ }^{1}(\mathrm{~kg})$ & 3 & 3 & 3 & 3 & 3 \\
Drèche (kg) & 6 & 6 & 6 & 6 & 6 \\
Huile de neem \% & 0 & 0,25 & 0,5 & 0,75 & 1 \\
Total & $\mathbf{1 0 0}$ & $\mathbf{1 0 0}$ & $\mathbf{1 0 0}$ & $\mathbf{1 0 0}$ & $\mathbf{1 0 0}$ \\
Composition nutritionnelle & & & & & \\
Energie métabolisable EM & & & & & \\
(Kcal/kg) & 3145,95 & 3146,33 & 3144,24 & 3144,97 & 3143,58 \\
Protéine brute & 19,72 & 19,75 & 19,74 & 19,77 & 19,83 \\
Matière grasse (\%) & 7,01 & 7,13 & 7,05 & 7,04 & 6,84 \\
Fibre brute (\%) & 5,28 & 5,36 & 5,4 & 5,43 & 5,42 \\
Lysine (\%) & 1,02 & 1,02 & 1,02 & 1,02 & 1,03 \\
Méthionine (\%) & 0,47 & 0,47 & 0,47 & 0,44 & 0,47 \\
Calcium (\%) & 0,65 & 0,65 & 0,65 & 0,65 & 0,64 \\
Phosphore (\%) & 0,49 & 0,5 & 0,51 & 0,52 & 0,52 \\
\hline
\end{tabular}

\section{RESULTATS}

Effet de l'huile de neem sur l'ingestion alimentaire (IA) des poulets de chair à croissance lente (g/poulet/jour)

L'Ingestion Alimentaire (IA) des poulets de chair a augmenté en fonction de l'âge des poulets pour les cinq rations (Tableau 2). L'IA des poulets soumis à l'alimentation contenant l'huile de neem a été légèrement inférieure à celle du lot témoin avec une différence significative à la dixième semaine $(\mathrm{P}<0,05)$.

Effet de l'huile de neem sur la croissance pondérale des poulets de chair en fonction de l'âge

Aucune différence significative n'a été obtenue entre les poids vifs moyens des poulets de différents traitements $(P>0,05)$, entre la cinquième et la huitième semaine (Figure 1). A partir de la neuvième semaine jusqu'à la douzième semaine, les lots $\mathrm{T} 0$ et $\mathrm{T} 0,50$ avaient une croissance pondérale similaire entre eux et statistiquement différente des autres lots. Le poids vif moyen le plus élevé $(1528 \pm 75,86 \mathrm{~g})$ a été obtenu chez les poulets de chair du lot $\mathrm{T} 0,50$.

Effet de l'huile de neem sur l'évolution du gain de poids moyen des poulets de chair à croissance lente au cours de la phase expérimentale (g/poulet/semaine).

L'évolution du gain de poids (Tableau 3) a montré que les poulets de chair du lot témoin et du lot T0,50 ont présenté un gain de poids plus élevé que les poulets de chair des groupes T0,25; T0,75 et T1. Cependant, la différence n'était pas significative entre les 
différents lots $(\mathrm{P}>0,05)$. A partir de la neuvième semaine, il y avait une différence significative entre les gains de poids en faveur des poulets de chair nourris avec la ration contenant $0,50 \%$ d'huile de neem $(247,2 \pm 78,2$ g).

Effet de l'huile de neem sur l'Indice de consommation (IC) des poulets de chair à croissance lente (g d'aliment/g de gain de poids)

Il n'y avait pas de différence significative $(P>0,05)$ entre les Indices de Consommation (IC) des poulets nourris avec la ration témoin et ceux nourris avec une alimentation contenant l'huile de neem (Tableau 4). Par ailleurs, une amélioration de l'IC a été observée chez les poulets de chair du lot T0,50 qui présentaient le plus bas IC. Sur l'ensemble de l'essai, l'IC le plus élevé a été obtenu avec le lot T0,75.

Effet de l'huile de neem sur le poids relatif des organes des poulets de chair

Les poids du pancréas, du gésier et du foie des poulets de chair des différents traitements n'ont montré aucune différence significative $(\mathrm{P}>0,05)$ (Tableau 5). Cependant, le poids du cœur des poulets soumis à une alimentation contenant l'huile de neem était significativement différent $(\mathrm{P}<0,05)$ de celui des poulets de chair du lot témoin.

Effet de l'huile de neem sur la longueur de l'intestin, le rendement en carcasse, le poids du bréchet et le $\mathrm{pH}$ ultime des poulets de chair

On a noté une différence significative entre les valeurs de la longueur de l'iléon entre les différents lots et il en ressort que les poulets du lot témoin (T0) avaient une longueur d'iléon plus court que celle des poulets soumis à l'alimentation contenant l'huile de neem (Tableau 6). Les rendements en carcasse au sein des différents lots ont montré une différence significative $(\mathrm{P}<0,05)$. Toutefois, les poulets du lot $\mathrm{T} 0,50$ avaient un rendement en carcasse plus élevé que celui des autres lots. Il n'y avait pas de différence significative (P> $0,05)$ entre les valeurs du poids du bréchet entre les lots, mais le lot témoin $\mathrm{T} 0$ avait le plus faible poids de bréchet.

Le $\mathrm{pH}$ ultime du filet des poulets de chair était similaire dans tous les traitements. Cependant, la viande des poulets du lot T0,75 a présenté un $\mathrm{pH}$ ultime légèrement plus faible.

Effet de l'huile de neem sur les paramètres biochimiques des poulets de chair

Les résultats ont montré une différence significative $(\mathrm{P}>0,05)$ entre les différents paramètres biochimiques étudiés au sein de différents lots de poulets de chair (Tableau 8). Cependant, on constate que la triglycéridémie, la protéinémie et l'albuminémie ont augmenté avec le niveau d'incorporation d'huile de neem dans la ration des poulets de chair. Quant à la glycémie, elle était légèrement plus élevée au sein du lot témoin sans une différence significative $(\mathrm{P}>0,05)$ avec les autres traitements.

Effet de l'huile de neem sur le coefficient d'utilisation digestive et métabolique des poulets de chair

L'ingestion de la matière sèche et de la protéine n'était pas significative entre les rations $(P>0,05)$ (Tableau 9). De plus, les taux de digestibilité de la matière sèche et de protéine brute étaient élevés dans tous les traitements. Néanmoins, les poulets de chair du lot T0,50 ont présenté un taux de digestibilité de matière sèche légèrement plus élevé $(92,35 \%)$ et le lot T1 un taux de digestibilité légèrement plus faible $(89,61 \%)$. Quant à la digestibilité de protéine brute, le lot T0,50 a présenté un taux de digestibilité plus élevé $(96,51 \%)$ que celui du lot T1 $(94,01 \%)$. Globalement, les coefficients d'utilisation digestive et métabolique de la matière sèche et de la protéine brute des poulets de l'ensemble des lots expérimentaux étaient similaires $(\mathrm{P}>0,05)$.

\section{Effet de l'huile de neem sur la mortalité}

Le taux de mortalité le plus important a été enregistré dans le lot témoin alors que le taux le plus faible a été enregistré dans les lots $\mathrm{T} 0,25$ et $\mathrm{T} 0,50$ (Figure 2). 
Tableau 2 : Effet de l'huile de neem sur l'ingestion alimentaire (IA) des poulets de chair à croissance lente (g/poulet/jour).

\begin{tabular}{lcccccc}
\hline & \multicolumn{5}{c}{ Traitements } & \\
\cline { 2 - 6 } Paramètres & T0 & T0,25 & T0,50 & T0,75 & T1 & p-value \\
\cline { 2 - 6 } IA 6 & $56,94 \pm 0,2$ & $55,17 \pm 1,46$ & $53,14 \pm 0,57$ & $55 \pm 1,01$ & $55,06 \pm 1,23$ & 0,13 \\
IA 7 & $60,09 \pm 0,49$ & $58,35 \pm 0,06$ & $56,29 \pm 0,86$ & $58,86 \pm 0,57$ & $59,2 \pm 0,80$ & 0,06 \\
IA 8 & $82,29 \pm 0,56$ & $80,99 \pm 1,02$ & $79,72 \pm 0,29$ & $79,83 \pm 0,23$ & $81,49 \pm 0,78$ & 0,2 \\
IA 9 & $92,13 \pm 0,90$ & $91,43 \pm 1,71$ & $90,58 \pm 0,29$ & $91,86 \pm 0,72$ & $92,43 \pm 0,86$ & 0,57 \\
IA 10 & $98,7 \pm 0,70^{\mathbf{a}}$ & $94,21 \pm 0,08^{\mathbf{b}}$ & $95,72 \pm 1,43^{\text {ab }}$ & $96,8 \pm 0,49^{\mathbf{a}}$ & $97,11 \pm 0,54^{\mathbf{a}}$ & 0,04 \\
IA 11 & $97,72 \pm 0,57$ & $96,79 \pm 1,61$ & $96,15 \pm 1,86$ & $97,43 \pm 0,86$ & $97,14 \pm 0,1$ & 0,95 \\
IA 12 & $96,86 \pm 0,29$ & $95,32 \pm 0,69$ & $95,14 \pm 20$ & $96,64 \pm 0,58$ & $96,75 \pm 0,68$ & 0,54 \\
IAm & $83,25 \pm 6,69$ & $81,75 \pm 6,75$ & $80,96 \pm 7,11$ & $82,49 \pm 7,01$ & $82,88 \pm 6,99$ & 0,99 \\
\hline
\end{tabular}

IA6, IA7, IA8, IA9, IA10, IA11, IA12 et IAm : ingestions alimentaires respectives de la sixième, septième, huitième, neuvième, dixième, onzième et douzième semaine et leur moyenne tout au long de la période expérimentale. ${ }^{\mathrm{a}, \mathrm{b}}$ Les moyennes sur la même ligne portant des lettres différentes étaient significativement différentes $(\mathrm{P}<0,05)$.

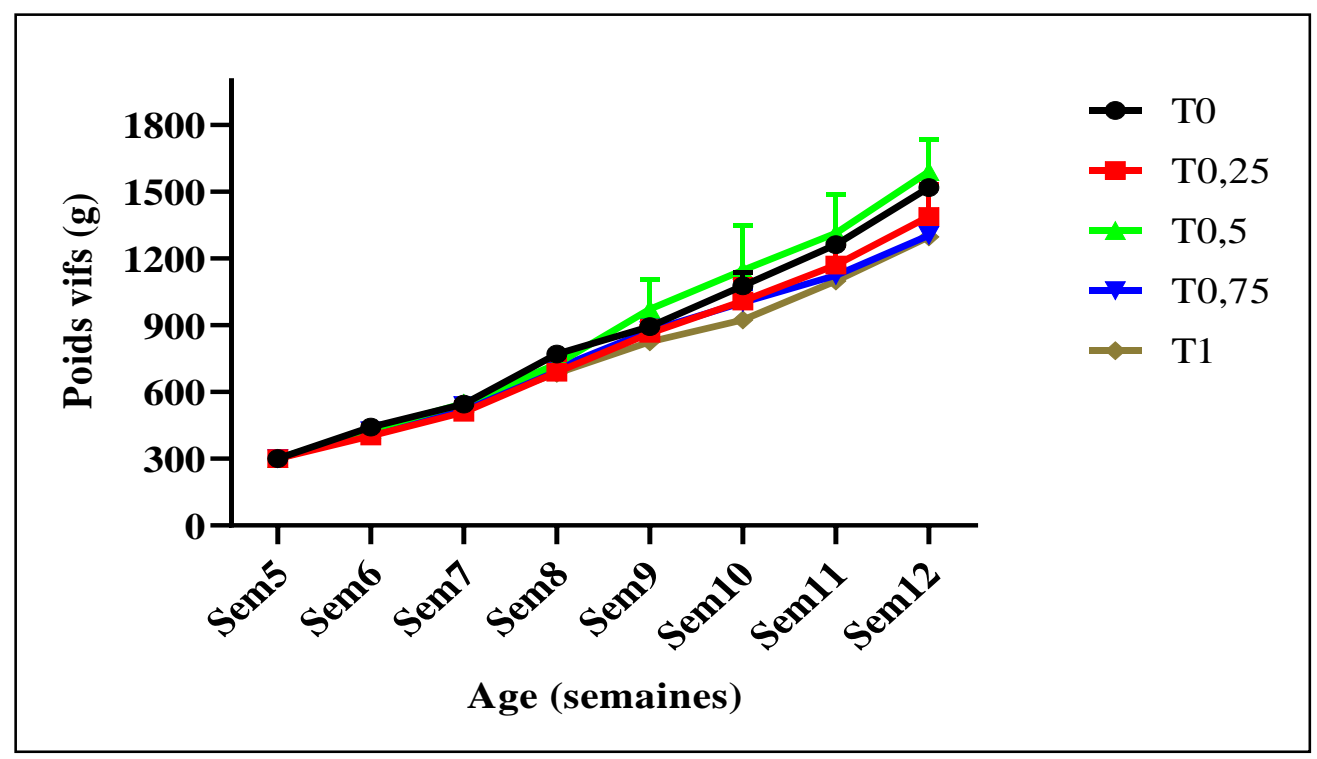

Figure 1: Effet de l'huile de neem sur la croissance pondérale des poulets de chair en fonction de l'âge. 
Tableau 3 : Effet de l'huile de neem sur l'évolution du gain de poids moyen des poulets de chair à croissance lente au cours de la phase expérimentale (g/sujet/semaine).

\begin{tabular}{lcccccc}
\hline & \multicolumn{7}{c}{ Lots } & \\
\cline { 2 - 6 } Paramètres & T0 & T0,25 & T0,50 & T0,75 & T1 & P-value \\
\hline GP 6 & $140,9 \pm 4,64$ & $101,3 \pm 31,74$ & $129,1 \pm 9,98$ & $124,6 \pm 4,58$ & $118,9 \pm 1,16$ & 0,47 \\
GP 7 & $103 \pm 10,64$ & $107,1 \pm 1,92$ & $119,1 \pm 2,74$ & $112,3 \pm 2,88$ & $113,8 \pm 4,215$ & 0,20 \\
GP 8 & $225,8 \pm 12,42$ & $180,2 \pm 9,65$ & $176,8 \pm 8,96$ & $168,3 \pm 30,87$ & $149,8 \pm 12,02$ & 0,22 \\
GP 9 & $123 \pm 16,96^{\mathbf{c}}$ & $174,7 \pm 0,42^{\mathbf{b}}$ & $247,2 \pm 78,2^{\mathbf{a}}$ & $168,5 \pm 0,65^{\mathbf{b}}$ & $142,9 \pm 11,71^{\text {bc }}$ & 0,04 \\
GP 10 & $182,4 \pm 32,63$ & $144,2 \pm 29,18$ & $174,6 \pm 47,31$ & $127,4 \pm 12,03$ & $98,17 \pm 40,24$ & 0,59 \\
GP 11 & $185,1 \pm 33,66$ & $162,6 \pm 6,56$ & $166,2 \pm 16,75$ & $122 \pm 32,13$ & $174,1 \pm 46$ & 0,83 \\
& & & & & & \\
GP 12 & $158,1 \pm 14,35$ & $118,1 \pm 41,35$ & $177 \pm 22,56$ & $79,34 \pm 6,51$ & $98,66 \pm 1,39$ & 0,22 \\
GPm & $159,75 \pm 17,90$ & $141,17 \pm 17,25$ & $170 \pm 26,64$ & $128,92 \pm 12,81$ & $128,03 \pm 16,68$ & 0,37 \\
\hline
\end{tabular}

GP6, GP7, GP8, GP9, GP10, GP11, GP12 et GPm: gain de poids respectifs de la sixième, septième, huitième, neuvième, dixième, onzième et douzième semaine et leur moyenne tout au long de la période expérimentale.

${ }^{\mathrm{a}, \mathrm{b}}$ Les moyennes sur la même ligne portant des lettres différentes étaient significativement différentes $(\mathrm{P}<0,05)$.

Tableau 4 : Effet de l'huile de neem sur l'Indice de consommation (IC) des poulets de chair à croissance lente ( $\mathrm{g}$ d'aliment/ $\mathrm{g}$ de gain de poids).

\begin{tabular}{ccccccc}
\hline & \multicolumn{5}{c}{ Lots } & \\
\cline { 2 - 6 } IC & T0 & T0,25 & T0,50 & T0,75 & T1 & P-value \\
\hline IC 6 & $3,7 \pm 0,09$ & $4,16 \pm 1,3$ & $2,96 \pm 0,23$ & $3,05 \pm 0,11$ & $3,2 \pm 0,03$ & 0,25 \\
IC 7 & $3,65 \pm 0,37$ & $3,48 \pm 0,06$ & $3,13 \pm 0,07$ & $3,32 \pm 0,08$ & $3,28 \pm 0,12$ & 0,23 \\
IC 8 & $3,53 \pm 0,18$ & $3,16 \pm 0,15$ & $3,23 \pm 0,15$ & $3,40 \pm 0,60$ & $3,88 \pm 0,32$ & 0,26 \\
IC 9 & $5,33 \pm 0,68$ & $3,63 \pm 0,11$ & $3,05 \pm 0,86$ & $3,85 \pm 0,01$ & $4,59 \pm 0,36$ & 0,07 \\
IC 10 & $3,74 \pm 0,57$ & $4,77 \pm 0,97$ & $3,8 \pm 1,05$ & $5,43 \pm 0,47$ & $6,42 \pm 3,49$ & 0,88 \\
IC 11 & $3,77 \pm 0,74$ & $4,17 \pm 0,23$ & $4,09 \pm 0,6$ & $6,03 \pm 1,7$ & $4,20 \pm 1,1$ & 0,88 \\
IC 12 & $4,18 \pm 0,39$ & $4,45 \pm 2,30$ & $3,9 \pm 0,63$ & $5,59 \pm 0,66$ & $4,86 \pm 0,14$ & 0,39 \\
ICm & $3,99 \pm 0,43$ & $3,97 \pm 0,73$ & $3,94 \pm 0,50$ & $4,38 \pm 0,52$ & $4,34 \pm 0,79$ & 0,42 \\
\hline
\end{tabular}

IC 6, IC 7, IC 8, IC 9, IC 10, IC 11, IC 12 et ICm : ingestion alimentaires respectives de la sixième, septième, huitième, neuvième, dixième, onzième et douzième semaine et leur moyenne tout au long de la période expérimentale. 
Tableau 5: Effet de l'huile de neem sur le poids des différents organes des poulets.

\begin{tabular}{|c|c|c|c|c|c|c|}
\hline \multirow[b]{2}{*}{ Paramètres } & \multicolumn{5}{|c|}{ Lots } & \multirow[b]{2}{*}{ Probabilité } \\
\hline & T0 & T0,25 & T0,50 & T0,75 & T1 & \\
\hline Pancréas (\%) & $0,36 \pm 0,04$ & $0,28 \pm 0,04$ & $0,32 \pm 0,04$ & $0,30 \pm 0,01$ & $0,29 \pm 0,06$ & 0,69 \\
\hline Gésier (\%) & $2,56 \pm 0,25$ & $2,30 \pm 0,16$ & $2,50 \pm 0,12$ & $2,43 \pm 0,13$ & $2,34 \pm 0,04$ & 0,41 \\
\hline Foie $(\%)$ & $2,31 \pm 0,20$ & $2,30 \pm 0,08$ & $2,41 \pm 0,27$ & $2,47 \pm 0,04$ & $2,45 \pm 0,10$ & 0,72 \\
\hline Cœur (\%) & $0,53 \pm 0,03^{a}$ & $0,43 \pm 0,02^{\mathbf{b}}$ & $0,44 \pm 0,02^{\mathbf{b}}$ & $0,43 \pm 0,04^{\mathbf{b}}$ & $0,41 \pm 0,02^{\mathbf{b}}$ & 0,03 \\
\hline
\end{tabular}

$\overline{a, b}$ Les moyennes sur la même ligne portant des lettres différentes étaient significativement différentes $(\mathrm{P}<0,05)$.

Tableau 6 : Effet de l'huile de neem sur la longueur de l'intestin des poulets de chair.

\begin{tabular}{lcccccc}
\hline & \multicolumn{5}{c}{ Lots } & \\
\cline { 2 - 5 } Paramètres & T0 & $\mathbf{T 0 , 2 5}$ & T0,50 & T0,75 & T1 & Probabilité \\
\hline $\begin{array}{l}\text { Longueur } \\
\text { duodénum } \\
(\mathrm{cm})\end{array}$ & $34,25 \pm 1,1$ & $35,75 \pm 0,8$ & $36,00 \pm 0,7$ & $32,63 \pm 1,1$ & $33,13 \pm 1,4$ & 0,19 \\
$\begin{array}{l}\text { Longueur } \\
\text { jéjunum }(\mathrm{cm})\end{array}$ & $63,75 \pm 3,1$ & $67,25 \pm 4,9$ & $68,50 \pm 0,6$ & $75,00 \pm 6,8$ & $59,25 \pm 1,7$ & 0,22 \\
$\begin{array}{l}\text { Longueur iléon } \\
(\mathrm{cm})\end{array}$ & $65,00 \pm 4,5^{\mathbf{c}}$ & $82,50 \pm 1,1^{\mathbf{a}}$ & $70,75 \pm 3,3^{\mathbf{c}}$ & $84,75 \pm 3,8^{\mathbf{a}}$ & $77,75 \pm 1,3^{\mathbf{b}}$ & 0,0074 \\
\hline $\begin{array}{l}\text { a.b } \\
\text { Les moyennes sur la même ligne portant des lettres différentes étaient significativement différentes (P }<0,05)\end{array}$ &
\end{tabular}

$\overline{\mathrm{a}, \mathrm{b}}_{\mathrm{b}}$ Les moyennes sur la même ligne portant des lettres différentes étaient significativement différentes $(\mathrm{P}<0,05)$.

Tableau 7: Effet de l'huile de neem sur le rendement en carcasse, le poids du bréchet et le $\mathrm{pH}$ ultime.

\begin{tabular}{lcccccc}
\hline & \multicolumn{5}{c}{ Lots } & \\
\cline { 2 - 6 } Paramètres & T0 & T0,25 & T0,50 & T0,75 & T1 & Probabilité \\
\hline Carcasse (\%) & $64,3 \pm 0,89^{\mathbf{b}}$ & $64,56 \pm 1,16^{\mathbf{b}}$ & $72,84 \pm 2,76^{\mathbf{a}}$ & $65,16 \pm 0,55^{\mathbf{b}}$ & $67,51 \pm 0,42^{\mathbf{b}}$ & $\mathbf{0 , 0 1}$ \\
Bréchet (\%) & $14,85 \pm 0,19$ & $15,19 \pm 1,23$ & $14,94 \pm 0,92$ & $15,65 \pm 1,38$ & $15,29 \pm 1,49$ & $\mathbf{0 , 9 9}$ \\
pHu & $5,68 \pm 0,01$ & $5,68 \pm 0,09$ & $5,71 \pm 0,05$ & $5,64 \pm 0,02$ & $5,73 \pm 0,05$ & $\mathbf{0 , 7 5}$ \\
\hline a,b Les moyennes sur la même ligne portant des lettres différentes étaient significativement différentes $(\mathrm{P}<0,05)$ & &
\end{tabular}


Tableau 8 : Effet de l'huile de neem sur les paramètres biochimiques des poulets de chair.

\begin{tabular}{|c|c|c|c|c|c|c|}
\hline \multirow[b]{2}{*}{ Paramètres } & \multicolumn{5}{|c|}{ Lots } & \multirow[b]{2}{*}{ Probabilité } \\
\hline & T0 & T0,25 & T0,50 & T0,75 & T1 & \\
\hline Triglycéride (mmol/l) & $0,16 \pm 0,06$ & $0,96 \pm 0,02$ & $1,1 \pm 0,86$ & $1,26 \pm 0,10$ & $1,32 \pm 0,10$ & 0,22 \\
\hline Protéine total (g/dl) & $2,86 \pm 0,28$ & $2,65 \pm 0,21$ & $3,24 \pm 0,36$ & $3,35 \pm 0,41$ & $3,46 \pm 0,10$ & 0,21 \\
\hline Albumine (g/dl) & $1,84 \pm 0,71$ & $1,85 \pm 0,32$ & $2,15 \pm 1,07$ & $2,28 \pm 0,22$ & $2,25 \pm 0,81$ & 0,20 \\
\hline Glucose (mmol/dl) & $22,04 \pm 3,38$ & $19,66 \pm 3,81$ & $18,53 \pm 3,18$ & $15,51 \pm 5,58$ & $14,35 \pm 6,57$ & 0,83 \\
\hline
\end{tabular}

Tableau 9 : Effet de l'huile de neem sur le coefficient d'utilisation digestive et métabolique des poulets de chair.

\begin{tabular}{|c|c|c|c|c|c|c|}
\hline \multirow[b]{2}{*}{ Paramètres } & \multicolumn{5}{|c|}{ Lots } & \multirow[b]{2}{*}{ Probabilité } \\
\hline & T0 & T0,25 & T0,50 & T0,75 & T1 & \\
\hline PB (g/kg d'aliment) & $19,54 \pm 0,27$ & $18,54 \pm 20$ & $19,8 \pm 0,25$ & $19,45 \pm 0,25$ & $18,89 \pm 0,10$ & 0,67 \\
\hline $\operatorname{CUDM}_{\mathrm{MS}} \%$ & $91,69 \pm 2,81$ & $90,71 \pm 3,30$ & $92,35 \pm 3,65$ & $90,3 \pm 1,70$ & $89,61 \pm 3,39$ & 0,39 \\
\hline $\mathrm{CUDM}_{\mathrm{PB}} \%$ & $95,08 \pm 0,07$ & $94,76 \pm 1,7$ & $96,51 \pm 1,49$ & $94,33 \pm 0,32$ & $94,01 \pm 3,51$ & 0,37 \\
\hline
\end{tabular}

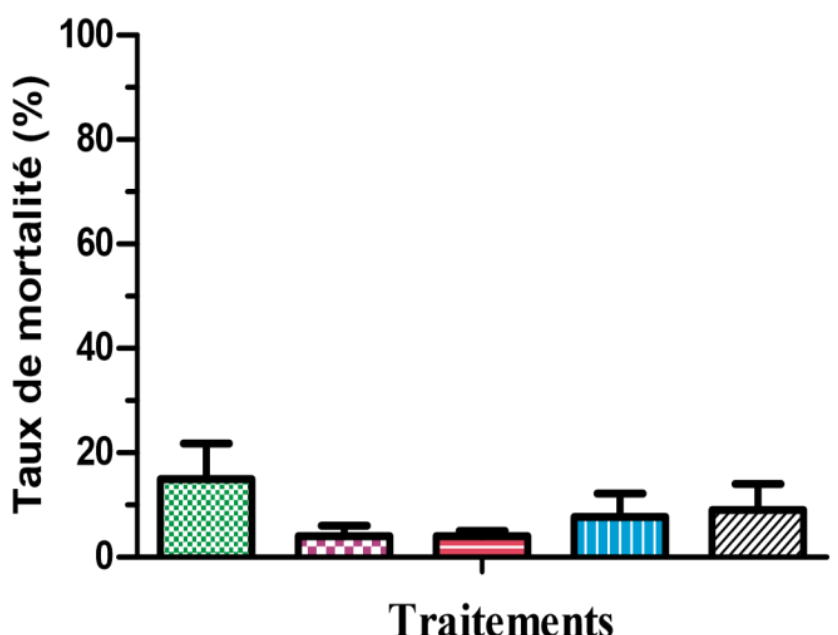

T0

$\mathrm{T} 0,25$

$\mathrm{T} 0,5$

Figure 2 : Variation du taux de mortalité des poulets de chair en fonction du traitement. 


\section{DISCUSSION}

Les valeurs de l'Ingestion Alimentaire chez les poulets de chair lors de cette expérimentation n'étaient pas en générale significativement différentes entre les lots témoins et les lots traités $(\mathrm{P}>0,05)$. Ceci montrerait qu'une ration contenant l'huile de neem permettrait aux poulets de chair de mieux moduler leur consommation alimentaire pour satisfaire leur besoin. Les travaux réalisés par Ingole et al. (2016) ont montré une diminution de la consommation alimentaire chez les poulets de chair en ajoutant $0,4 \%$ d'huile de neem à leur alimentation. Les travaux de Jeffri et al. (2010) ont également rapporté qu'en augmentant les sources de lipides dans l'alimentation des poulets de chair, la quantité d'aliment ingérée diminue et l'efficacité de l'alimentation est améliorée. Globalement, les poids vifs moyens et le gain de poids des poulets de chair dans les différents lots étaient similaires $(P>0,05)$. Ceci était sans doute lié au Coefficient d'Utilisation Digestive et Métabolique de la matière sèche et de la protéine qui était élevé dans ce lot. Cela suggèrerait donc une amélioration du gain de poids et du poids vif moyen des poulets de chair à un taux de $0,5 \%$ d'huile de neem. Ces résultats sont conformes à ceux d'Onyimonyi et al. (2009), qui ont montré une amélioration du poids vif et de la prise de poids lors de l'ajout de feuilles de neem en poudre au régime alimentaire des poulets de chair à un taux de 0,5\%. Par contre Guerreiro Neto et al. (2011) ont montré que l'utilisation d'huile de soja, de graisse de volaille et de leur mélange dans le régime alimentaire des poulets de chair n'influençait pas les performances de croissance, en l'occurrence le poids vif. Selon ces auteurs, cette différence serait due aux facteurs antinutritionnels de l'huile de soja, à la souche et à l'âge des sujets utilisés.

Une légère augmentation de l'IC a été observée au sein des lots traités avec la ration contenant l'huile de neem sauf le lot T0,50, ce qui a montré que les poulets de chair de ce lot auraient mieux digéré l'aliment entrainant un gain de poids contrairement à ceux des autres lots. Ces résultats ne sont pas en concordance avec ceux rapportés par Paul et al. (2020) qui ont montré qu'il y a une amélioration de l'IC des poulets de chair soumis à une alimentation contenant l'extrait de feuille de neem à $0,1 \%$. Selon Vanessa et al. (2019), cette différence serait due au fait que l'huile de neem renferme plusieurs composés bioactifs aux propriétés antimicrobiennes et anti protozoaires qui peuvent limiter la croissance et la colonisation des espèces bactériennes non pathogènes à une certaine dose.

Les poids relatifs de la plupart des organes internes n'ont pas été affectés $(\mathrm{P}>0,05)$ par l'incorporation de l'huile de neem dans la ration des poulets de chair. Cependant, le poids relatif du cœur a été significativement différent $(\mathrm{P}<0,05)$ entre les lots. Et il est ressorti que le poids relatif du cœur des poulets traités était plus petit que celui du cœur du lot témoin. Ces résultats confirment ceux de Paul et al. (2020) qui ont observé des variations significatives du poids des organes internes en l'occurrence le cœur des poulets de chair nourris avec un extrait de feuilles de neem. Cette différence serait due aux caractéristiques physicochimiques de l'huile de neem qui contient plusieurs composés bioactifs, à l'âge et à la souche des sujets utilisés. De plus, ces résultats ont montré que la longueur de l'iléon des poulets de chair était significativement plus élevée lorsque le régime contenait l'huile de neem, ce qui se traduirait par une meilleure absorption/digestion des nutriments et un poids carcasse significativement élevée chez ces poulets. En effet l'efficacité de l'absorption serait influencée par la surface disponible pour le passage des nutriments, plus l'intestin est long, plus les villosités sont nombreuses, meilleure est l'absorption (Yang et al., 2013). Ces résultats corroborent avec ceux trouvés par Seidavi et al. (2013) qui ont rapporté que la longueur de l'iléon des poulets de chair nourris avec trois sources d'huile (Suif, huile de colza et huile de tournesol) à un taux de $4 \%$ était significativement différente $(\mathrm{p}<0,05)$. Toutefois cette différence pourrait être liée à la composition ou au type de graisse utilisée.

La longueur du tractus intestinal qui était plus élevée chez les sujets soumis à l'alimentation contenant l'huile de neem a mis en lumière les meilleurs rendements de la 
carcasse obtenus au sein de ces lots. En effet Benatmane et Bouacem (2014) ont obtenu un meilleur rendement de la carcasse des poulets de chair soumis avec une alimentation contenant de la matière grasse, en l'occurrence une ration enrichie en huiles végétales (olive et colza) à 4\%. Par contre, Sese et al. (2013) ont montré que le rendement de la carcasse était similaire pour les poulets de chair nourris à des niveaux gradués de Mucuna utilis. La qualité de la viande des poulets de chair peut être rapidement estimée en déterminant la valeur du pH des muscles de bréchet (Glamoclija et al., 2015). Dans cette étude, les valeurs du $\mathrm{pH}$ ultime des filets de poulets de chair étaient comprises entre 5,64 et 5,73. Santos et al. (2005) et Berri et al. (2007), ont également trouvés des $\mathrm{pH}$ plus bas $(\mathrm{pH}<6)$ chez les génotypes à croissance lente. Fosoul et al. (2018) ont rapporté que ces valeurs basses du $\mathrm{pH}$ seraient dues aux battements des ailes des animaux sur la ligne d'abattage affectant ainsi le métabolisme musculaire. D'après Fanatico et al. (2005) et Bouvarel et al. (2008), ces valeurs sont liées à une réserve de glycogène dans les bréchets au moment de l'abattage des animaux. Les taux de mortalité obtenus pourraient être dus aux propriétés antimicrobiennes et anti-parasitaires de l'huile de neem, ce qui a contribué à réduire la contamination microbienne et à améliorer l'efficacité alimentaire des oiseaux (Hasanain et al., 2018). Les profils sanguins et les performances de croissance constituent des indices importants de l'état physiologique des animaux. En effet, les teneurs sériques en protéines, en triglycérides et en albumine dans les lots traités ont augmenté avec le niveau d'huile de neem dans le régime alimentaire. Shihab et al. (2017), ont également montré qu'il y a une légère augmentation de la concentration en protéines totales, triglycérides et en albumine en ajoutant la poudre de feuilles de neem au régime des poulets de chair à raison de $2 \mathrm{~g} / \mathrm{kg}$. De même Addass et al. (2012) ont également rapporté que les paramètres biochimiques sériques des poulets de chair pouvaient varier en fonction de plusieurs facteurs, notamment l'alimentation, l'âge, la race, le sexe, l'état physiologique et la conduite de l'élevage. Le taux de glucose a diminué au sein des lots traités mais pas significativement par rapport au témoin. Ce qui serait probablement dû à une diminution de la néoglucogenèse suite à l'incorporation de l'huile de neem dans leur ration (Kebbaj 2012). Par ailleurs les valeurs de CUDM obtenues ont montré que les poulets de chair ont digéré efficacement les aliments. Ces résultats sont conformes à ceux de Cruz et al. (2005) qui ont trouvé des taux de digestibilité de la matière sèche qui variaient entre $(87,13-92,67 \%)$ et ceux de la protéine brute qui étaient entre (93,84 - 97,94\%) en comparant la ration complète au système cafétéria. La fraction digestible de l'alimentation est la partie de l'aliment ingéré qui ne se retrouve pas dans les fèces (Atchade et al., 2019). Cette amélioration de la digestibilité pourrait être due à la dégradation de la matière sèche et de protéine brute dans le tube digestif à la fois par les enzymes digestives et de la microflore des poulets de chair.

\section{Conclusion}

$\mathrm{Au}$ terme de cette étude qui avait pour but d'évaluer l'effet de l'huile de neem sur les performances zootechniques, les caractéristiques de la carcasse et les paramètres biochimiques sériques des poulets de chair à croissance lente, il en ressort que les poulets de chair soumis à une ration contenant l'huile de neem ont présenté une longueur de l'intestin plus élevée et un poids du cœur plus faible que le groupe témoin. L'utilisation de l'huile de neem dans l'alimentation aurait exercé un effet favorable sur les performances de production des poulets de chair. Ainsi son utilisation exercerait un effet optimal à un taux de $0,5 \%$ dans l'alimentation des poulets de chair.

\section{CONFLIT D'INTERETS}

Les auteurs déclarent qu'il n'existe pas de conflit d'intérêts.

\section{CONTRIBUTIONS DES AUTEURS}

KKK et TT ont conceptualisé l'étude. MB, KKK et YTK ont collecté et analysé les spécimens. KKK a interprété les données et écrit le manuscrit. TT a révisé le manuscrit de 
manière critique par rapport au contenu intellectuel. Tous les auteurs ont contribué à la préparation de l'article et approuvé la version soumise.

\section{REMERCIEMENTS}

Les auteurs adressent leurs sincères remerciements et leur profonde gratitude à tous ceux qui ont contribué à la réalisation du présent article.

\section{REFERENCES}

Addass PA, David DL, Edward A, Zira KE, Midau A. 2012. Effect of Age, Sex and Management System on Some haematological parameters of intensively and semi-intensively kept chicken in Mubi, Adamawa State, Nigeria. Iranian Journal of Applied Animal Science, 2 (3): 277-282. DOI:

https://www.sid.ir/en/journal/ViewPaper .aspx?id=268179

Atchade GST, Houndonougbo FM, Chrysostome CAAM, Mensah GA. 2019. Digestibility of feeds in broiler chicken (Galus galus linnaeus, 1758) in Africa: a review. Int. J. Biol. Chem. Sci., 13(2): 1127-1139.

DOI: https://dx.doi.org/10.4314/ijbcs.v13i2.43

Benatmane F, Bouacem K. 2014. Impact de l'enrichissement de l'aliment en huiles végétales (olive et colza) à hauteur de $4 \%$ sur les performances de croissance du poulet de chair. Nutr. Clin. Metab., 1(28) : 67-68, DOI: 10.1016/S09850562(14)70645-4.

Berri CM, Debut V, Sante L, houtellier C, Arnould B, Boutten N, Sellier E, Baeza N, Jehl Y, Jego MJ, Duclos J, Le BihanDuval E. 2007. Variations in chicken breast meat quality; implications of struggle and muscle glycogen content at death. Br. Poult. Sci., 46: 572-579. DOI: https://doi.org/10.1080/00071660500303 099.

Bouvarel I, Chagneau AM, Lescoat P, Tesseraud S, Leterrier C. 2008. Fortyeight-hour cycle sequential feeding with diets varying in protein and energy contents: adaptation in broilers at different ages. Poult. Sci., 87: 196-203. DOI: https://doi.org/10.3382/ps.200700205.

Cruz VC, Pezzato AC, Pinheiro DF, Gonçalves JC, Sartori JR. 2005. Effect of free-choice freeding on the performance and ileal digestibility of nutrients in broilers. Braz. J. Poult. Sci., 3: 143-150. DOI : http://www.redalyc.org/articulo.oa?id=179 713988002

Diedhiou D. 2017. Fractionnement analytique de la graine de neem (Azadirachta indica A. Juss.) et de la graine de dattier du désert (Balanites aegyptiaca L.) Valorisation des constituants de la graine de neem par bioraffinage. Thèse Doc, Université Cheikh Anta Diop de Dakar (UCAD), Sénégal, p. 298.

Fanatico AC, Cavitt LC, Pillai PB, Emmert JL, Owens CM. 2005. Evaluation of slowergrowing broiler genotypes grown with and without outdoor access: Meat quality. Poult. Sci., 84: 1785-1790. DOI : 10.1093/ps/84.11.1785.

FAO. 2018. Production de volaille en Afrique de l'ouest. (http://www.fao.org/docrep/013/a1706e/ al706e00.pdf)

FAO. 2019. Situation géographique et conditions climatiques de Lomé (Togo). fao.org/docs/pdf/Tog192042.pdf

FAOSTAT. 2020. FAO Statistical Yearbook, Number of poultry in Togo, Retrieved September.

http://www.fao.org/faostat/fr/\#home

Faye M. 2010. Nouveau procédé de fractionnement de la graine de neem (Azadirachta indica A. Juss) sénégalais : production d'un bio pesticide d'huile et de tourteau. Thèse Doc, Université de Toulouse, France, p. 266.

Fosoul AS, Azarfar S, Gheisari A, Khosravinia AH. 2018. Energy utilisation of broiler chickens in response to guanidinoacetic acid supplementation in diets with various energy contents. Br. J. Nutr., 120(2): $131-140 . \quad$ DOI: $10.1017 / \mathrm{S} 0007114517003701$.

Glamoclija N, Starcevic M, Janjic J, Ivanovic J, Boskovic M, Djordjevic J, Markovic R, 
Baltic MZ. 2015. The effect of breed line and age on measurements of $\mathrm{pH}$-value as meat quality parameter in breast muscles (m. pectoralis major) of broiler chickens. Procedia Food Sci., 5: 89 - 92. DOI: 10.1016/j.profoo.2015.09.023

Guerreiro Neto AC, Pezzato AC, Sartori JR, Mori C, Cruz VC, Fascina VB, Pinheiro DF, Madeira LA, Gonçalvez JC. 2011. Emulsifier in Broiler Diets Containing Different Fat Sources. Braz. J. Poult. Sci., 13(2): 119-125. DOI: $10.1590 / \mathrm{S} 1516-$ 635X2011000200006

Hasanain NE, Suha SA, Hasan SJ. 2018. A review on the effects of neem (Azadirachta indica) as feed additive in poultry production. J Entomol Zool Stud, 6(1): $\quad 1331-1333 . \quad$ DOI: http://doi.org/55941772

Ingole RS, Patil RM, Pathak VP, Joshi MV. 2016. Effect of Neem Oil supplementation on growth Performance and Hematobiochemical Profile in Broilers. The Indian J. Vet. Sci. Biotechnol., 2: 33-38. DOI: http://dx.doi.org/10.21887/ijvsbt.v12i2.3 739

Inoussa KY, Charles P, Marius KS, Bréhima D, Mamoudou HD. 2020. Caractéristiques physicochimiques de quelques matières premières utilisées dans la formulation des aliments pour volaille au Burkina Faso. J. Appl. Biosci., 151: 15598 15604.

DOI: https://doi.org/10.35759/JABs.151.8

Jahanzeb A, Sohail HK, Ahsan H, Muhammad Y. 2012. Effect of the levels of Azadirachta indica dried leaf meal as phytogenic feed additive on the growth performance and haemato-biochemical parameters in broiler chicks. J. Appl. Anim. Res., 40: 336-345. DOI: https://doi.org/10.1080/09712119.2012.6 92329

Jeffri D, Firman H, Kamyab A. 2010. Comparison of soybean oil with an animal/vegetable blend at four energy levels in broiler rations from hatch to market. Int. Poult. Sci., 9: 1027-1030. DOI : 10.3923/ijps.2010.1027.1030
Kebbaj RE. 2012. Base moléculaire des effets de l'huile d'argan sur le métabolisme mitochondrial et peroxysomal des acides gras et sur l'inflammation. Thèse Doc., Université de Bourgogne, Maroc, p. 87.

Koona S, Budida S. 2011. Antibacterial potential of the extracts of the leaves of Azadirachta indica linn. Not. Sci. Biol., 3 : 65-69.

DOI: https://doi.org/10.15835/nsb315470

Makeri HK, Maikai VA, Nok JA. 2007. Effect of topical application of neem seed (Azadirachta indica) extract on sheep infested with Amblyomma variegatum. Afr. J. Biotechnol., 6: 2324-2327. DOI: 10.5897/AJB2007.000-2364

Nahimana G, Ossebi W, Missohou A, Ayssiwede SB. 2019. Analyse de l'importance socio-économique de l'aviculture familiale dans le Département de Salemata au Sénégal. Int. J. Biol. Chem. Sci., 13(7): 3131-3143. DOI :

https://dx.doi.org/10.4314/ijbcs.v13i7.13

Novidzro KM, Wokpor K, Fagla BM, Koudouvo K, Dotse K, Osseyi E, Koumaglo KH. 2019. Etude de quelques paramètres physicochimiques et analyse des éléments minéraux, des pigments chlorophylliens et caroténoïdes de l'huile de graines de Griffonia simplicifolia. Int. J. Biol. Chem. Sci., 13(4): 2360-2373. DOI :

https://dx.doi.org/10.4314/ijbcs.v13i4.38

Ogbuewu IP, Okoro VM, Okoli IC, Iloeje MU. 2011. Evaluation of dried leaf meal of an ethnomedicinal plant-neem on linear growths and reproductive tract morphometry of rabbit does. Elec. J. Env. Agricult. Food Chem., 10: 2153-2159. DOI:

https://www.cabdirect.org/cabdirect/abst ract $/ 20113159302$

Onyimonyi AE, Olabode A, Okeke GC. 2009. Performance and economic characteristics of broilers fed varying dietary levels of neem leaf meal (Azadirachta indica). Int. J. Poult. Sci., 8: 256-259.

DOI: 10.3923/ijps.2009.256.259 
Paul TK, Hasan MM, Haque MA, Talukder S, Sarker YA, Sikder MH, Khan M, Sakib MN, Kumar A. 2020. Dietary supplementation of Neem (Azadirachta indica) leaf extracts improved growth performance and reduced production cost in broilers. Vet. World, 13(6): 1050-1055. DOI: www.doi.org/10.14202/vetworld

Ponka R, Goudoum A, Tchungouelieu AC, Fokou E. 2016. Evaluation nutritionnelle de quelques ingrédients entrant dans la formulation alimentaire des poules pondeuses et porcs d'une ferme d'élevage au Nord-Ouest Cameroun. Int. J. Biol. Chem. Sci., 10(5): 2073-2080. DOI : http://dx.doi.org/10.4314/ijbcs.v10i5.11

Santos AL, Sakomura NK, Freitas ER, Fortes CMLS, Carrilho ENVM, Fernandes JBK. 2005. Growth, performance, carcass yield and meat quality of three broiler chickens strains. Rev. Bras. Zootec., 34: 15891598.

DOI :

https://doi.org/10.1590/1678-416210316

Seidavi A, Laudadio V, Tufarelli V. 2013. Influence of dietary fat source on growth performance responses and carcass traits of broiler chicks. Asian Australas J. Anim. Sci., 5: 705-710. DOI: 10.5713/ajas.2012.12633

Sese BT, Okpeku M, Geroge OS. 2013. Organ Weights, Carcass Characteristics and Blood Chemistry of Broiler Birds Fed Graded Levels of Mucuna Utilis Leave. J Vet Adv., 3(4): 146-152. DOI: 10.5455/jva.20130510062334

Shihab IM, AL-Zuhariy MT, Abdullah SM, Mutar SS. 2017. Impact of supplementation Neem powder (Azadirachta indica) to diet broiler in immunological, physiological and productive traits. Adv. Environ. Biol, 11(3): 44-51. DOI: https://doi.org/209481492

Sonaiya EB, Swan SEJ. 2004. Production en aviculture familiale: un manuel technique. Organisation des Nations Unies pour l'Alimentation et l'agriculture, FAO/Productions et Santé Animales, Rome, $134 \mathrm{p}$.

Tossou ML, Houndonougbo MF, Abiola FA, Chrysostomec AAM. 2014. Comparaison des performances de production et de la qualité organoleptique de la viande de trois souches de poulets chair (hubbard, cobb et ross) élevées au bénin. Revue CAMES de Science de la Vie, de la Terre et Agronomie, 02: 30-35. DOI: http://www.lecames.org/spip.php?article 1

Valarmathy K, Gokulakrishnan M, Kausar MS, Paul KA. 2010. Study of antimicrobial activity of ethanolic extracts of various plant leaves against selected microbial species. Int. J. Pharm. Sci. Res., 1: 293295.

DOI: https://citeseerx.ist.psu.edu/viewdoc/dow nload?doi=10.1.1.1067.8265\&rep=rep1 \&type $=$ pdf

Vanessa MS, Jean RK, Nguepi DK. 2019. Effects of graded levels of Azadirachta indica seed oil on growth performance and biochemical profiles of broiler chickens. Vet. Med. Sci., 1: 1-9. DOI: 10.1002/vms3.162

Windisch WM, Schedle K, Plitzner C, Kroismayr A. 2008. Use of phytogenic products as feed additives for swine and poultry J. Anim. Sci., 86: E140-148. DOI : $10.2527 /$ jas.2007-0459. 